\title{
Hybrid halobismuthates: structure and optical properties
}

\author{
Petr A. Buikin ${ }^{1,4}$, Vitalii Yu. Kotov ${ }^{2,4}$, Alexander A. Korlyukov ${ }^{3}$, Andrey B. llyukhin ${ }^{4}$ \\ ${ }^{1}$ D. Mendeleev University of Chemical Technology of Russia, 125047, Moscow, Russian Federation; \\ ${ }^{2}$ National Research University Higher School of Economics, 101000, Moscow, Russian Federation; \\ ${ }^{3}$ Institute of Organoelement Compounds of RAS, 119991, Moscow, Russian Federation;
}

${ }^{4}$ N. S. Kurnakov Institute of General and Inorganic Chemistry, Russian Academy of Sciences, 119991, Moscow, Russian Federation;

$$
\text { peterzzz@mail.ru }
$$

The chemistry of hybrid halometallates attracting increasing interest of researchers. The interest is associated with a number of physical properties inherent in this class of compounds, such as semiconductivity, photochromism, luminescence, etc. One of the prominent representatives of this class are hybrid halosmuthates - promising candidates for solar energy.

The crystal engineering of halobismuthates to prepare the substances with given anion seems to be the highest priority. In this work we report the synthesis and crystal structure of organic-inorganic hybrid halobismuthates of 1,4'-bipyridine cations. The structure and optical properties of the isolated compounds were studied. The novel anion $\left[\mathrm{Bi}_{6} \mathrm{I}_{26}\right]^{6-}$ was discovered in the structure $[\mathrm{PyPy}]_{2}[\mathrm{PyPyH}]_{2} \mathrm{Bi}_{6} \mathrm{I}_{26}$. The DFT calculation of this structure showed the total energy of six Bi-I interactions in BiI 6 polyhedra remains almost constant for all crystallographically independent bismuth atoms. In this case the correlation between Bi-I bond length values and interaction energy values $\left(\mathrm{R}^{2}=0.9993\right)$ was performed. Using this correlation, the statistical analysis of Bi-I bond energies in 262 iodine-bismuthate anions found in the CCDC database (ver 5.40 September 2019) was performed. The total energy of six Bi-I bonds in $\mathrm{BiI}_{6}$ polyhedra forming various iodine-bismuthate anions does not depend on the structure of the anions was shown. The data suggest the main factor affecting the formation of the final structure of hybrid iodobismuthates is not the energy benefit of the formation of one form or another of a bismuth-containing anion, but a combination of weak intermolecular interactions. In this fact the synthesis of such compounds with a given anion is impossible.

Keywords: hybrid halobismuthates, crystal structure, DFT calculations, crystal engineering 